\title{
Stress concentration due to an array or hemispherical cavities at the surface of an elastic half-space
}

\author{
XIAOPING LU†, J.R. BARBER* and MARIA COMNINOU \\ Department of Mechanical Engineering and Applied Mechanics, University of Michigan, Ann Arbor, \\ MI 18109, USA ("author for correspondence)
}

Received 27 November 1989; in revised form 19 March 1991

\begin{abstract}
The paper investigates the perturbation in an otherwise uniform stress field in an elastic half-space due to a doubly-periodic array of small hemispherical holes at the free surface. The solution is obtained using three potential functions of double Fourier series form in Galerkin's strain potential solution, the coefficients of which are determined using the collocation method. The unperturbed field is taken to be one of uniform plane stress parallel to the free surface. Two special cases are studied - uniform tension and uniform shear stress. Numerical results for these cases can be generalized by superposition to give solutions for a general state of biaxial plane stress. It is found that, for both tension and shear, the maximum stress concentration occurs at the bottom of the holes. The stress concentration factor increases with the ratio of hole spacing to radius, approaching the known solution for a single hemispherical hole at large ratios.
\end{abstract}

\section{Introduction}

Many engineering materials have various kinds of surface defects such as blow holes, scratches and microcracks, which tend to intensify the local stress field and hence reduce the load carrying capacity of the component. Such defects can act as initiation sites for fatigue cracks and are therefore an important factor in the experimentally observed sensitivity of fatigue life to surface finish. Blow holes, in particular, are difficult to avoid in the manufacturing of components from resin-based composite materials, because of the necessity for mixing of the resin and its relatively high viscosity which inhibits the mobility of air bubbles in the solidifying component. Experimental evidence also indicates that surface defects influence the propagation of the fracture surface in such materials [1].

The perturbation in a state of uniform biaxial hydrostatic tension due to a single hemispherical surface cavity was analysed by Eubanks [2] and results for more general spheroidal shapes have been given by Fujita $e t$ al. $[3,4]$. However, the problem of the interaction between the stress fields around

\footnotetext{
$\dagger$ Now at Department of Mathematics, University of Wollongong, NSW 2500, Australia.
} 
several cavities has not been addressed and forms the subject of the present paper. Specifically, we investigate the perturbation in an otherwise uniform biaxial state of plane stress due to a periodic array of identical hemispherical cavities at the surface of an elastic half-space. Results are presented for the cases where the unperturbed field is one of uniaxial tension or pure shear, since more general loadings can then be constructed using superposition.

The perturbed stress fields are represented in terms of Galerkin strain potentials, which are chosen in the form of double Fourier series. The periodic properties of the series permit us to define the boundary conditions in terms of the surface tractions in a unit cell containing a single hole. The collocation method is used to satisfy the boundary conditions at the free surface of the half-space and at the hole surface.

\section{Method of solution}

We consider the elastic isotropic half-space, $z>0$ in which a state of uniform stress is perturbed by a periodic array of small hemispherical holes of radius $a$ at the surface $z=0$ as shown in Fig. 1. The centers of the holes are situated

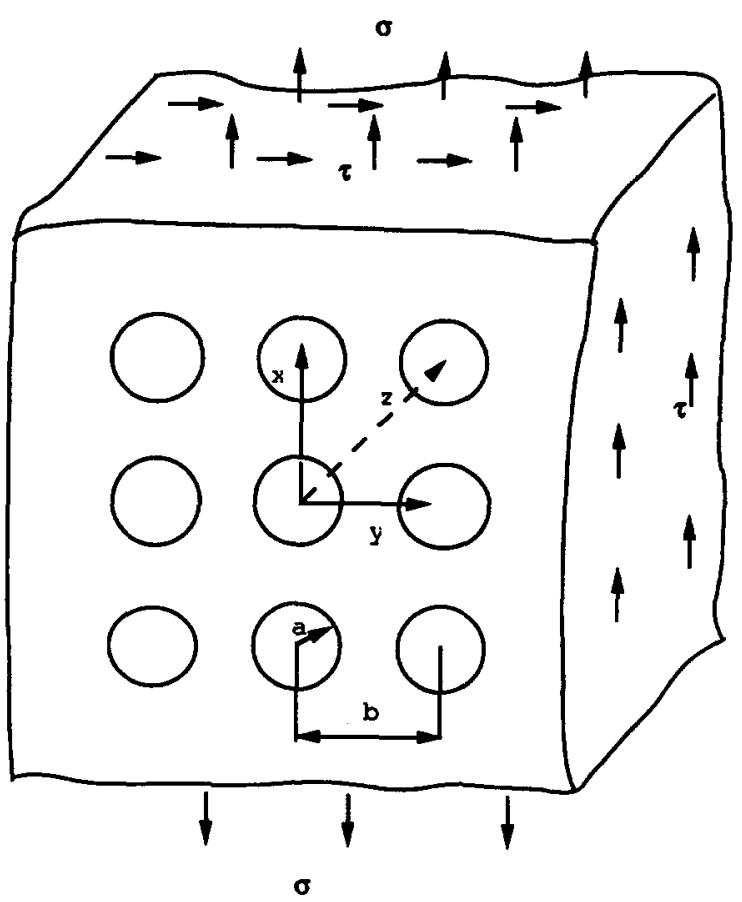

Fig. 1. Configuration of the system. 
at the points $(m b, n b, 0)$, where $m=0, \pm 1, \pm 2 \ldots ; n=0, \pm 1, \pm 2, \ldots$ We assume that the holes do not intersect each other and hence that $b>2 a$.

The boundary conditions at infinity take the form

$$
\sigma_{x x} \rightarrow \sigma, \sigma_{y y}, \sigma_{z z}, \sigma_{x y}, \sigma_{z x} \rightarrow 0, \quad z \rightarrow \infty
$$

for tensile loading, and

$$
\sigma_{x y} \rightarrow \tau, \sigma_{x x}, \sigma_{y y}, \sigma_{z z}, \sigma_{y z}, \sigma_{z x} \rightarrow 0, \quad z \rightarrow \infty
$$

for shear loading.

We also require that the surface of the holes and the remaining parts of the surface $z=0$ be traction-free. These conditions can be stated in the form

$$
\begin{aligned}
& \sigma_{z x}=\sigma_{z y}=\sigma_{z z}=0, \quad(x \pm m b)^{2}+(y \pm n b)^{2}>a^{2}, \quad z=0, \\
& \sigma_{R \theta}=\sigma_{R \phi}=\sigma_{R R}=0, \quad(x \pm m b)^{2}+(y \pm n b)^{2}+z^{2}=a^{2}, \quad z>0
\end{aligned}
$$

where $(R, \theta, \phi)$ defines a set of spherical polar coördinates centered on the hole in question.

We construct the solution of the problem in the form

$$
[S]=[U]+\left[S_{0}\right]
$$

where $[U]$ is the unperturbed state of uniform stress and $\left[S_{0}\right]$ is a corrective solution, describing the perturbation in $[U]$ due to the array of holes.

\section{Potential function representation}

Solution $\left[S_{0}\right]$ is represented in terms of the Galerkin potential

$$
\begin{aligned}
& \nabla^{4} \mathbf{F}=-\frac{\mathbf{K}}{1-v}, \\
& \sigma_{x x}=2(1-v) \frac{\partial \nabla^{2} X}{\partial x}+\left(v \nabla^{2}-\frac{\partial^{2}}{\partial x^{2}}\right) \operatorname{div} \mathbf{F}, \\
& \sigma_{x y}=(1-v)\left(\frac{\partial \nabla^{2} X}{\partial y}+\frac{\partial \nabla^{2} Y}{\partial x}\right)-\frac{\partial^{2}}{\partial x \partial y} \operatorname{div} \mathbf{F},
\end{aligned}
$$

etc., where $\mathbf{F}=X \mathbf{i}+Y \mathbf{j}+Z \boldsymbol{k}$ is the Galerkin vector, $\boldsymbol{K}$ is the body force and 
$v$ is Poisson's ratio for the material. The remaining stress components can be obtained from equations $(7,8)$ by cyclic permutation of $X, Y, Z$ and $x, y, z$. Since there is no body force in the present problem, equation (6) reduces to

$$
\nabla^{4} \mathbf{F}=0,
$$

and hence $\mathbf{F}$ is required to be a biharmonic vector function.

Westergaard [5 Art. 70] argues that the component $Z$ of the Galerkin vector can always be arbitrarily set to zero without loss of generality. However, his proof depends upon the construction of a biharmonic function $f$ such that

$$
\frac{\partial^{2} f}{\partial x \partial z}=-Z
$$

and there are restrictions on the domains for which this can be done. This difficulty was first noted by Sokolnikoff [6] in the related problem of completeness of the Papcovich-Neuber solution when one component of the corresponding vector function is set to zero. A more detailed investigation of the question is given by Eubanks and Sternberg [7] who show that the corresponding integration can be performed as long as every line parallel to the $z$ axis intersects the boundary of the body in at most two points. This condition is satisfied in the present problem, thus permitting us to set $Z$ to zero.

A further simplification in the representation can be achieved by writing

$$
X=f_{1}+z f_{2} ; \quad Y=f_{3}+z f_{4}
$$

where the functions $f_{i}$ are harmonic [8]. It then follows that the harmonic parts $f_{1}, f_{3}$ of $X, Y$ can be replaced by a single harmonic strain potential

$$
\phi=-\frac{\partial f_{1}}{\partial x}-\frac{\partial f_{3}}{\partial y}
$$

in terms of which

$$
\sigma_{x x}=\frac{\partial^{2} \phi}{\partial x^{2}}, \quad \sigma_{x y}=\frac{\partial^{2} \phi}{\partial x \partial y}, \text { etc. }
$$

(see [5, Art. 67]).

In summary, the solution $\left[S_{0}\right]$ is represented by superposing a Galerkin vector

$$
\mathbf{F}_{0}=z\left(\mathbf{i} f_{2}+\mathbf{i} f_{4}\right)
$$

and a strain potential $\phi$, where $f_{2}, f_{4}, \phi$ are harmonic functions of $x, y, z$ and 
the corresponding stress components are defined through equations $(7,8)$ for $F_{0}$ and (13) for $\phi$. We notice that this representation gives us three independent harmonic functions and permits us in general to satisfy three traction boundary conditions at the free surface.

The doubly periodic nature of the problem and the requirement that the perturbed field decay as $z \rightarrow \infty$ shows that the appropriate form for the functions $\phi, f_{2}, f_{4}$ is

$$
\sum_{i=0}^{\infty} \sum_{j=0}^{\infty} A_{i j} \mathrm{e}^{-\lambda \sqrt{i^{2}+j^{2} z}} \cos \sin (i \lambda x) \cos \sin (j \lambda y)
$$

where $\lambda=2 \pi / b, A_{i j}$ are a set of arbitrary constants to be determined from the boundary conditions and the choice of sine and cosine in the various series depends upon the symmetry of the applied loading. These functions are harmonic for all $A_{i j}$ and the choice of a negative argument for the exponentials ensures that the perturbed field decays as $z \rightarrow \infty$ as required by $(1,2)$. It is also easily verified that the use of functions like $(15)$ in $(7,8,13,14)$ generates stress fields which are doubly periodic, as required. ${ }^{1}$

The periodicity and symmetry of the problem and the representation permits us to satisfy the boundary conditions by applying $(3,4)$ inside the unit cell $0<x<b / 2,0<y<b / 2$. We also note that on the hemispherical surface $x^{2}+y^{2}+z^{2}=a^{2}, x>0$, it is more convenient to replace the boundary conditions (4) by the equivalent conditions

$$
\left.\begin{array}{l}
\sigma_{x x} n_{x}+\sigma_{x y} n_{y}+\sigma_{x z} n_{z}=0 \\
\sigma_{y x} n_{x}+\sigma_{y y} n_{y}+\sigma_{y z} n_{z}=0 \\
\sigma_{z x} n_{x}+\sigma_{z y} n_{y}+\sigma_{z z} n_{z}=0
\end{array}\right\}
$$

where $n_{x}=-x / a, n_{y}=-y / a, n_{z}=-z / a$ are the direction cosines of the spherical surface.

By imposing the boundary conditions $(3,16)$ at a suitable set of collocation points, we obtain a set of algebraic equations for the unknown coefficients in the series (15), from the solution of which the stress components can be obtained, using equations $(7,8,13,14)$.

\section{Uniaxial tensile loading}

When the applied loading consists of uniaxial tension (equation (1)), the problem is symmetrical about the planes $x=0, y=0$ and the appropriate

\footnotetext{
${ }^{1}$ This would not be true if for example the Papcovich-Neuber solution were used, which is the reason for preferring the present representation.
} 
double series forms derived from equations $(14,15)$ are

$$
\begin{aligned}
& X=\sum_{i=1}^{\infty} \sum_{j=0}^{\infty} A_{i j} z \mathrm{e}^{-\lambda \sqrt{i^{2}+\beta^{2}} z} \sin (i \lambda x) \cos (j \lambda y), \\
& Y=\sum_{i=0}^{\infty} \sum_{j=1}^{\infty} B_{i j} z \mathrm{e}^{-\lambda \sqrt{i^{2}+\rho^{2}} z} \cos (i \lambda x) \sin (j \lambda y), \\
& \phi=\sum_{i=0}^{\infty} \sum_{j=0}^{\infty} C_{i j} \mathrm{e}^{-\lambda \sqrt{i^{2}+j^{2} z}} \cos (i \lambda x) \cos (j \lambda y),
\end{aligned}
$$

where $A_{i j}, B_{i j}, C_{i j}$ are the unknown constants to be determined from the boundary conditions $(3,16)$.

To obtain a numerical solution, we truncate the series at $i, j=N$, giving a total of $N(3 N+4)$ unknown constants. We therefore require an equal number of algebraic equations, which we obtain by enforcing conditions $(3,16)$ at a set of collocation points chosen as the zeros of the first neglected trigonometric functions in the double series [9] which fall within the unit cell $0<x<b / 2,0<y<b / 2$, i.e.

$$
\begin{aligned}
& \sin \lambda(N+1) x_{l} \cos \lambda(N+1) y_{l}=0, \quad l=1,2, \ldots, N(N+1), \\
& \cos \lambda(N+1) x_{m} \sin \lambda(N+1) y_{m}=0, \quad m=1,2, \ldots, N(N+1), \\
& \cos \lambda(N+1) x_{n} \cos \lambda(N+1) y_{n}=0, \quad n=1,2, \ldots,(N+1)^{2},
\end{aligned}
$$

\section{Shear loading}

When the unperturbed stress field consists of pure shear (equation (2)), the problem is antisymmetric about $x=0, y=0$ and the following potential functions are appropriate:

$$
\begin{aligned}
& X=\sum_{i=0}^{\infty} \sum_{j=1}^{\infty} A_{i j} z \mathrm{e}^{-\lambda \sqrt{i^{2}+j^{2}} z} \cos (i \lambda x) \sin (j \lambda y) ; \\
& Y=\sum_{i=1}^{\infty} \sum_{j=0}^{\infty} B_{i j} z \mathrm{e}^{-\lambda \sqrt{i^{2}+j^{2}} z} \sin (i \lambda x) \cos (j \lambda y) ; \\
& \phi=\sum_{i=1}^{\infty} \sum_{j=1}^{\infty} C_{i j} \mathrm{e}^{-\lambda \sqrt{i^{2}+j^{2}} z} \sin (i \lambda x) \sin (j \lambda y) .
\end{aligned}
$$

Truncating the series at $i, j=N$, we obtain $N(3 N+2)$ unknown constants, which are determined by imposing boundary conditions $(3,16)$ at the collocation points

$$
\cos \lambda(N+1) x_{l} \sin \lambda(N+1) y_{l}=0, \quad l=1,2, \ldots, N(N+1) ;
$$




$$
\begin{aligned}
& \sin \lambda(N+1) x_{m} \cos \lambda(N+1) y_{m}=0, \quad m=1,2, \ldots, N(N+1) ; \\
& \sin \lambda(N+1) x_{n} \sin \lambda(N+1) y_{n}=0, \quad n=1,2, \ldots, N^{2} .
\end{aligned}
$$

\section{Results and discussion}

Numerical calculations have been performed for several values of the ratio $b / a$ in the two cases of applied uniaxial tension and pure shear. Solutions were obtained for various values of the number of terms $N$ in the truncated series and the results showed less than $1 \%$ change in the stress components for increase in $N$ beyond a value between 4 and 10, depending on the type of loading and the ratio $b / a$. Notice incidentally that a value of $N=10$ results in a system of 340 unknowns and equations in the case of uniaxial tension. A value of Poisson's ratio of 0.3 was used throughout the calculations.

Figure 2 shows the distribution of maximum principal stress at the plane $z=a$ for the case of uniform tensile loading in the $x$ direction and $b / a=4$. The stress is greatest near the hole, reaching a maximum of $2.08 \sigma$ at the bottom of the hole $(0,0, a)$. At this point, the stress components $\sigma_{x x}, \sigma_{y y}$ are principal stresses (as required by symmetry), the former being large and tensile, whilst the later is small and compressive.

The stress concentration factor $\sigma_{\max } / \sigma$ is shown in Fig. 3 and varies from 1.44 to 2.20 as $b / a$ increases from 2.4 to 5 . Over the same range, the dimensionless orthogonal stress $\sigma_{y y}$ is compressive, varying monotonically from -0.091 to -0.205 . At $b / a=2$, the edges of adjacent holes would just touch. The present method is not well-suited to this limiting case, but the trend of the results indicates that the stress concentration factor would fall to close to unity and the orthogonal stress to zero - a result which could be predicted from considerations of force flow, since the remaining 'islands' between the holes would then have little influence on the stress field in the material below the plane $z=a$.

At the other extreme, as $b / a \rightarrow \infty$, the problem reduces to that of the single hole in a field of uniaxial tension. Eubanks [2] obtained a stress concentration factor of 2.23 for the related problem of a single hole in biaxial hydrostatic tension, but Fujita et al. claim that a more accurate result is 2.18 . The present results can be compared with these solutions by superposing equal unixial tensile fields in the two orthogonal directions $x, y$, and predict a stress concentration factor of 2.00 at $b / a=5$.

Figure 4 shows the largest principal stress $\sigma_{x x}$ along the axis of symmetry of the hole, $x=y=0$, for $b / a=4$. The stress concentration diminishes rapidly with increasing $z$ falling to the unperturbed value at about $z=2 a$. Similar results were found for other ratios $b / a$. 


\section{MAXIMUM PRINCIPAL STRESS \\ TENSILE LOADING}

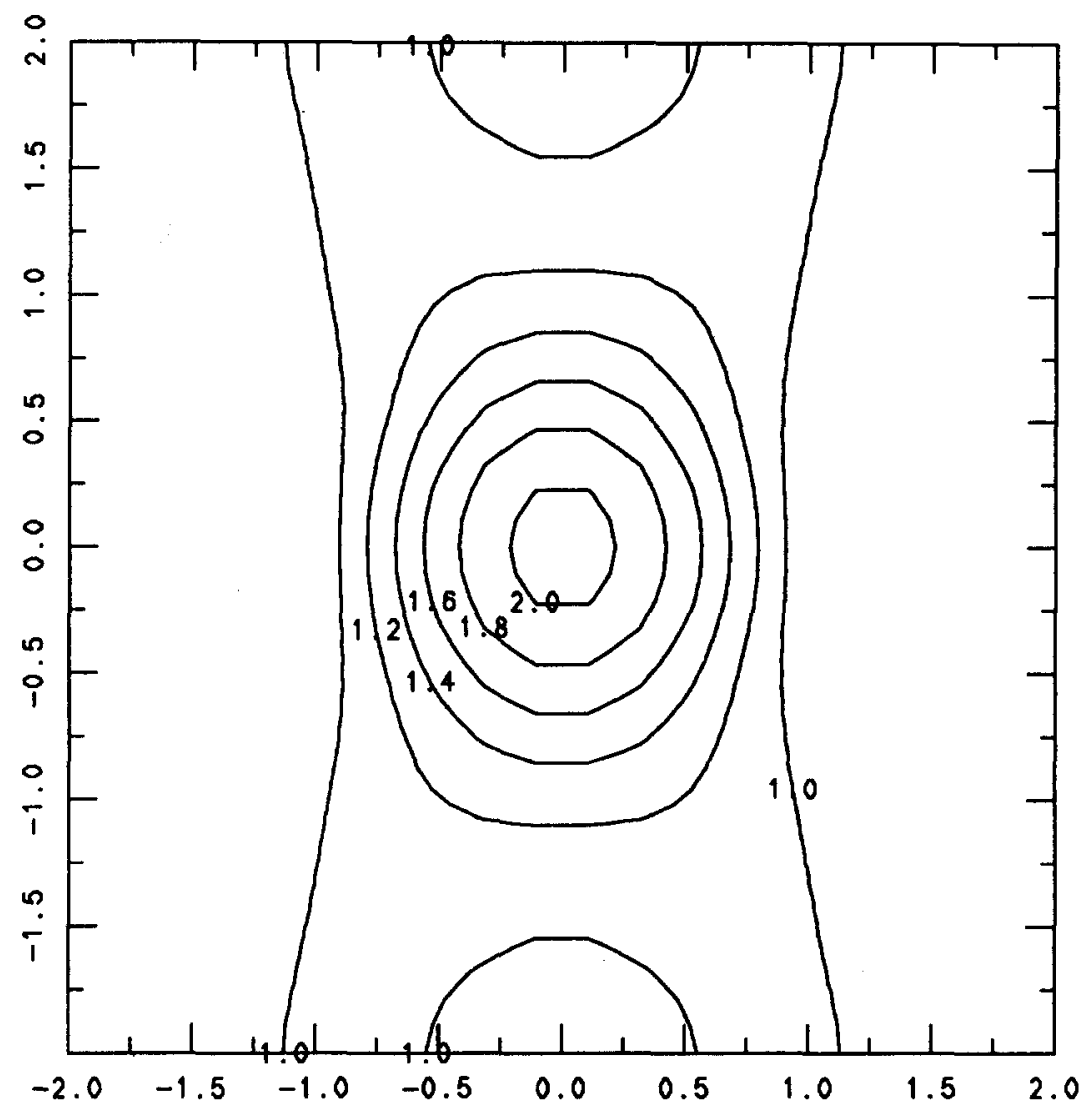

Fig. 2. Contour plot of maximum principal stress on $z=a$ for uniaxial tensile loading $(b / a=4)$.

Corresponding results for the case of pure shear loading are shown in Figs. 5-7. Figure 5 shows the maximum principal stress on the plane $z=a$ for $b / a=4$. The maximum stress again occurs at the bottom of the hole $(0,0, a)$ and consists of a state of pure shear, with $\sigma_{x y}$ the only non-zero stress component.

The stress concentration factor $\left(\sigma_{x y}\right)_{\max } / \tau$ is shown in Fig. 6 as a function of $b / a$. As in the case of tension, the results increase monotonically with $b / a$, and suggest a value of 3.10 for the single hole $(b / a \rightarrow \infty)$. At the other extreme, they approach an intuitive limit of unity as $b / a \rightarrow 2$. Notice incidentally that the stress concentration in shear for the single hole can be obtained by superposing orthogonal states of uniaxial tension and compression, but the 


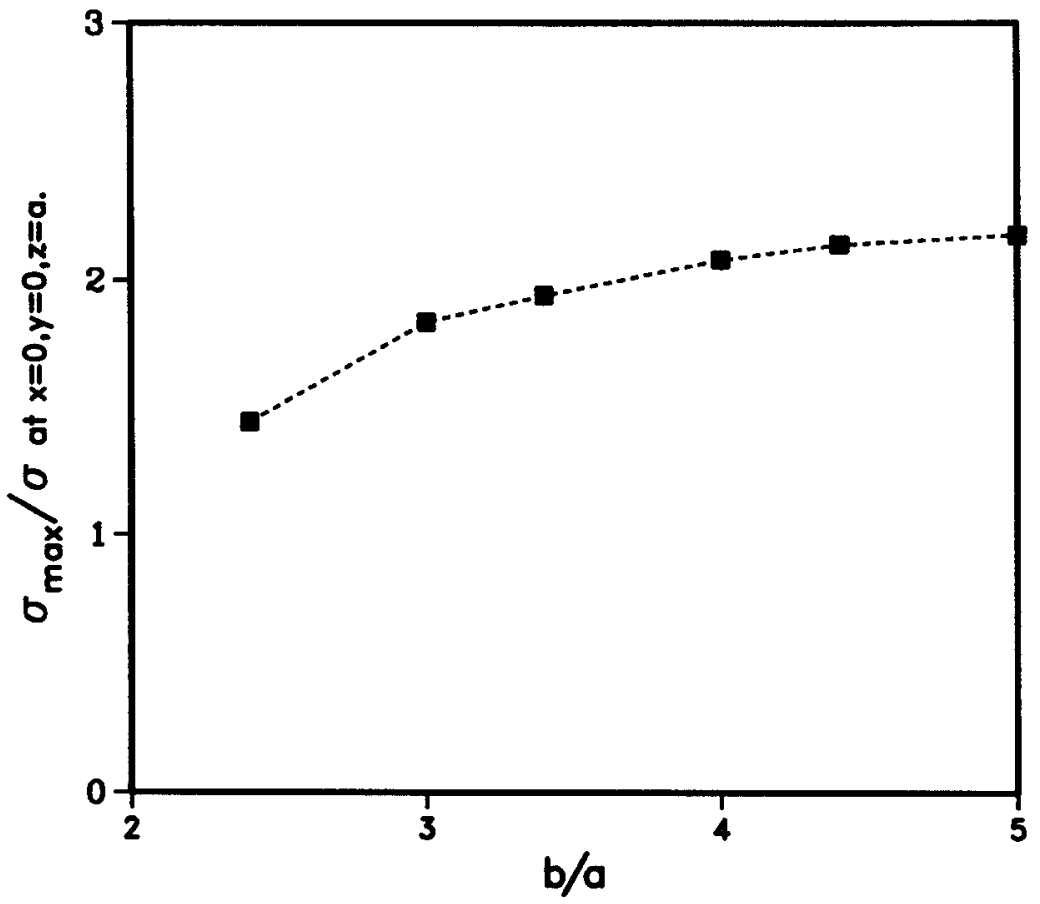

Fig. 3. Maximum principal stress at the bottom of the hole $(0,0, a)$ vs. $b / a$, for uniaxial tension.

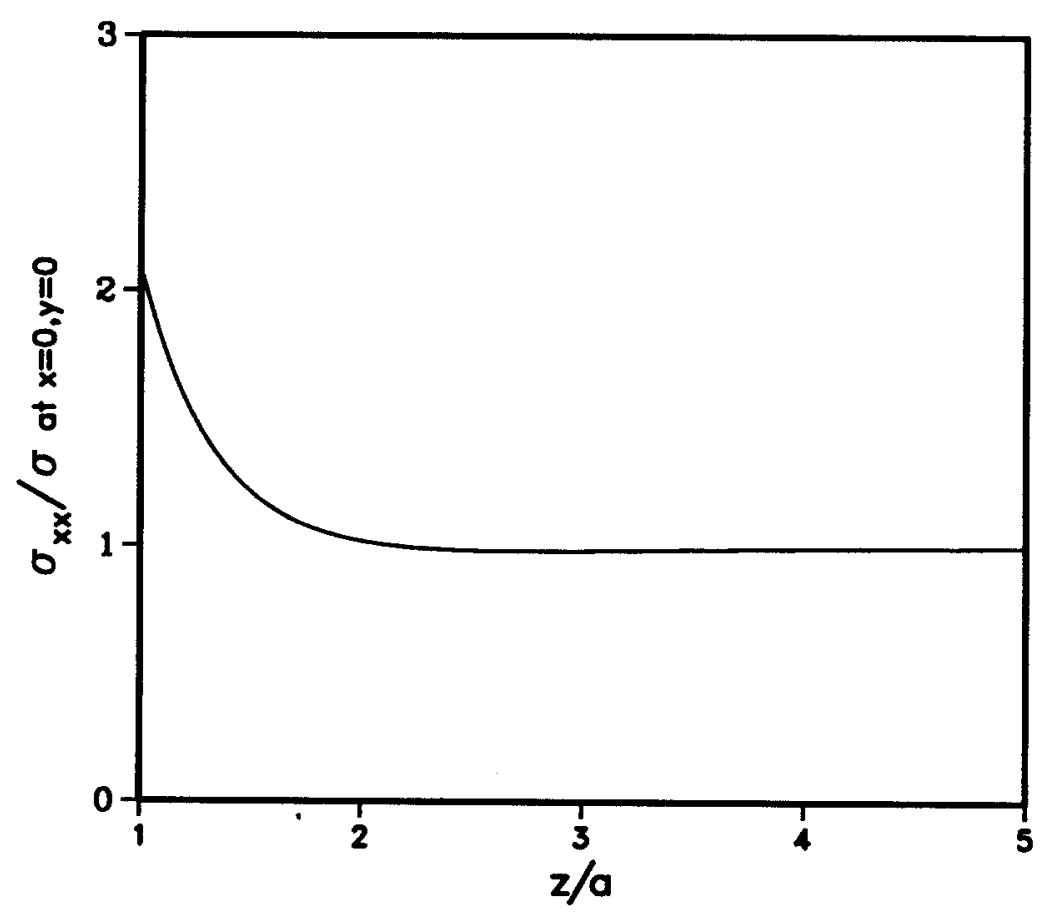

Fig. 4. Decay of $\sigma_{x x}$ along the $z$ axis for uniaxial tensile loading $(b / a=4)$. 
SHEAR LOADING

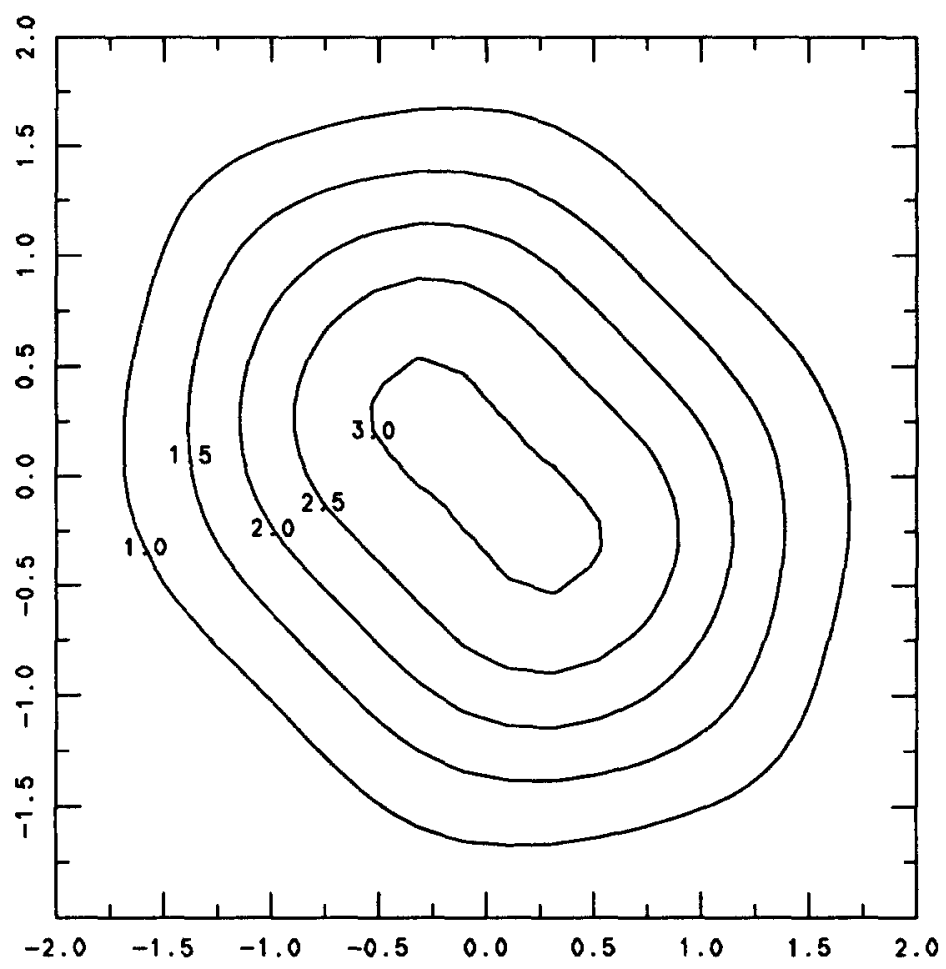

Fig. 5. Contour plot of maximum principal stresses on $z=a$ for shear loading $(b / a=4)$.

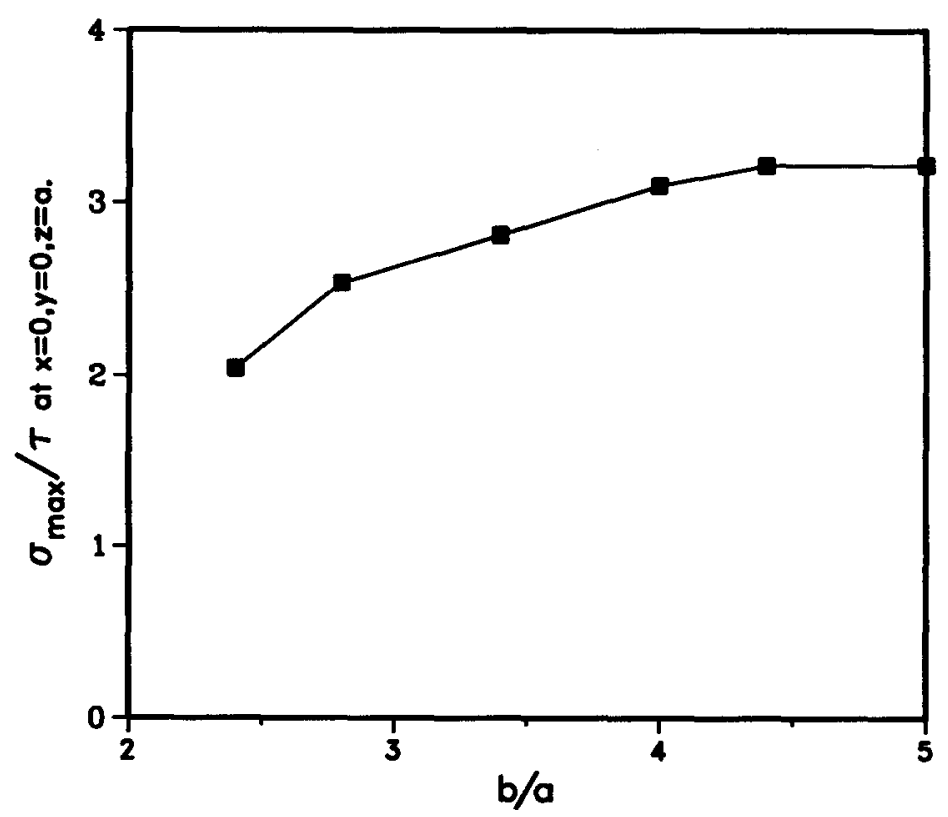

Fig. 6. Shear stress at the bottom of the hole $(0,0, a)$ vs. $b / a$, for shear loading. 


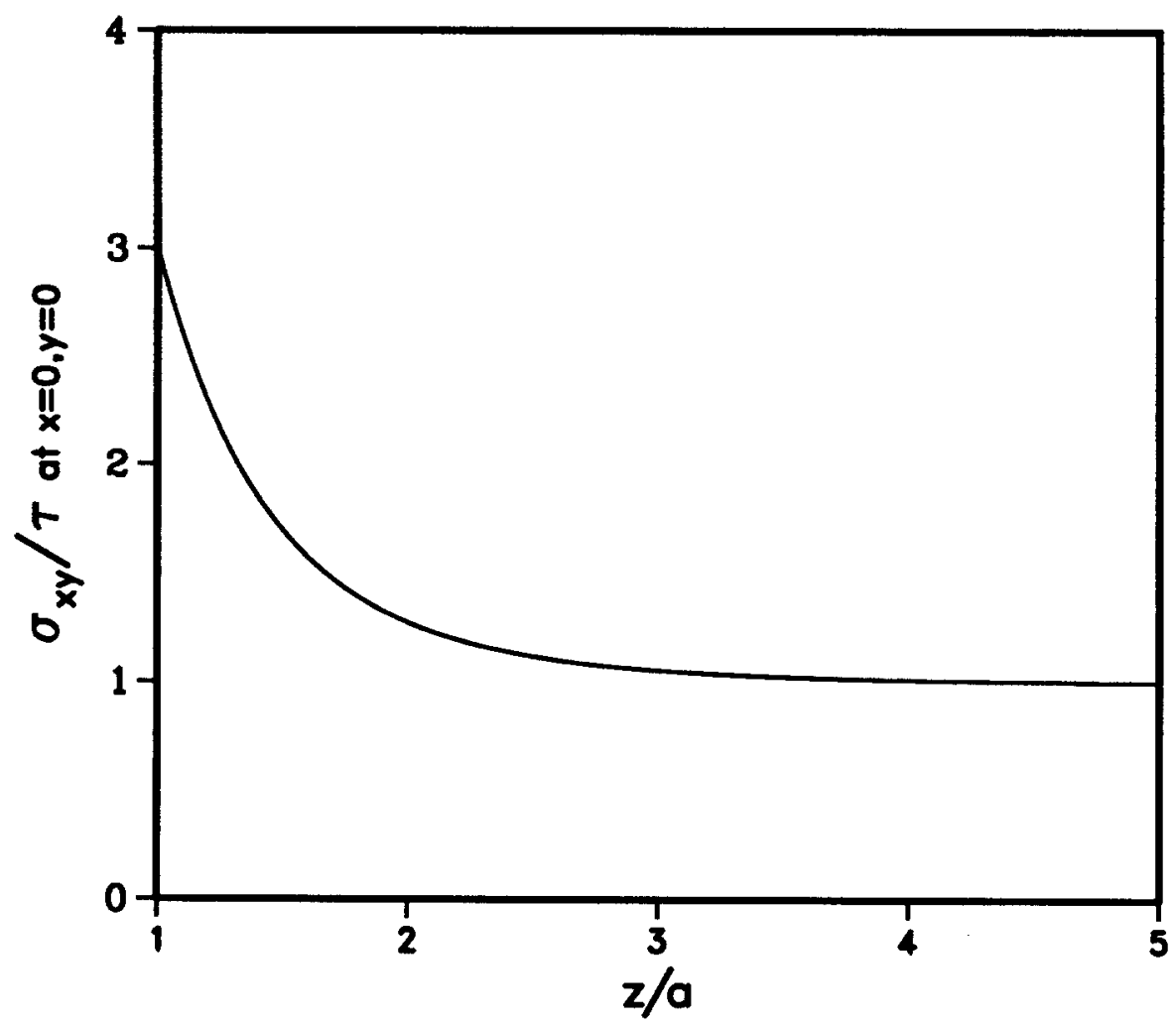

Fig. 7. Decay of $\sigma_{x y}$ along the $z$ axis for shear loading $(b / a=4)$.

same superposition cannot be used for finite $b / a$, since the array of holes renders the body geometrically anisotropic.

Finally Fig. 7 shows the decay of $\sigma_{x y} / \tau$ along the line $x=y=0$ for $b / a=4$.

\section{Conclusions}

The results show that the maximum stress concentration due to the array of holes always occurs at the bottom of the hole and the magnitude of the stress concentration factor decreases monotonically from the single hole value when $b / a$ is large to a value near unity (no stress concentration) when the holes nearly touch. Previously published results for a single hole in a biaxial hydrostatic tensile field give a good approximation (better than $10 \%$ ) when $b / a>5-i$.e. when the space between the holes is greater 1.25 times their diameter. In all cases, the stresses decay rapidly with depth to the applied uniform value. 


\section{References}

1. Maria Comninou, An overview of interface cracks. Eng. Frac. Mech. 37 (1990) 197-208.

2. R.A. Eubanks, Stress concentration due to a hemispherical pit at a free surface. $A S M E J$. Appl. Mech. 21 (1954) 57-62.

3. T. Fujita, E. Tsuchida and I. Nakahara, Stress concentration due to a hemiprolate spheroidal pit at a free surface of a semi-infinite body under all-around tension. Bull. Japan Soc. Mech. Eng. 23 (1980) 1048-1054.

4. T. Fujita, E. Tsuchida and I. Nakahara, On the stress concentration around a hemi-oblate spheroidal pit in a semi-infinite body. Bull. Japan Soc. Mech. Eng. 24 (1981) 15-21.

5. H.M. Westergaard, Theory of Elasticity and Plasticity. New York: Dover (1952), Chapter 6.

6. I.S. Sokolnikoff, Mathematical Theory of Elasticity. New York: McGraw-Hill, 2nd. edn. (1965).

7. R.A. Eubanks and E. Sternberg, On the completeness of the Boussinesq-Papcovich stress functions. Rat. Mech. Anal. 5 (1956) 734-756.

8. Y.C. Fung, Foundations of Solid Mechanics. Englewood Cliffs: Prentice-Hall, 2nd. edn. (1965), pp. 207-208.

9. J.P. Boyd, Private communication. 